\title{
Buckling of an elastic ridge: competition between wrinkles and creases
}

\author{
C. Lestringant, ${ }^{1}$ C. Maurini, ${ }^{1}$ A. Lazarus, ${ }^{1}$ and B. Audoly ${ }^{2}$ \\ ${ }^{1}$ Sorbonne Universités, UPMC Univ Paris 06, CNRS, \\ UMR 7190, Institut $\delta$ 'Alembert, F-75005, Paris, France \\ ${ }^{2}$ Laboratoire de Mécanique des Solides, CNRS, UMR 7649, \\ Département de Mécanique, École Polytechnique, 91128 Palaiseau CEDEX, France
}

\begin{abstract}
We investigate the elastic buckling of a triangular prism made of a soft elastomer. A face of the prism is bonded to a stiff slab that imposes an average axial compression. We observe two possible buckling modes which are localized along the free ridge. For ridge angles $\phi$ below a critical value $\phi^{\star} \approx 90^{\circ}$ experiments reveal an extended sinusoidal mode, while for $\phi$ above $\phi^{\star}$ we observe a series of creases progressively invading the lateral faces starting from the ridge. A numerical linear stability analysis is set up using the finite-element method and correctly predicts the sinusoidal mode for $\phi \leq \phi^{\star}$, as well as the associated critical strain $\epsilon_{\mathrm{c}}(\phi)$. The experimental transition at $\phi^{\star}$ is found to occur when this critical strain $\epsilon_{\mathrm{c}}(\phi)$ attains the value $\epsilon_{\mathrm{c}}\left(\phi^{\star}\right)=0.44$ corresponding to the threshold of the sub-critical surface creasing instability. Previous analyses have focused on elastic crease patterns appearing on planar surfaces, where the role of scale-invariance has been emphasized; our analysis of the elastic ridge provides a different perspective, and reveals that scale-invariance is not a sufficient condition for localization.
\end{abstract}

PACS numbers: 46.32.+x, 46.70.De, 83.80.Va

In extended systems, elastic instabilities generally produce smooth patterns having a well-defined wavelength. There are numerous examples involving an elastic beam [1] or a thin film 2, 4] on an elastic foundation, a bulk elastic material with inhomogeneous elastic properties [5], or rod-like solids with large incompatible strain [6] 8 , with applications ranging from morphogenesis [1] to the active control of surface properties [9]. An important exception to this rule is when the bifurcation problem has no intrinsic length-scale as happens for a compressed hyperelastic block, a problem considered by Biot [10, 11: a continuum of linear modes appear simultaneously at the bifurcation threshold with all possible wavelengths. This free-surface instability has been characterized numerically and experimentally only recently, and was found to be subcritical, localized and non-linear in essence [12 20]. In spite of recent progress [21, 22, there is no simple and systematic theoretical argument that explains why and in which circumstances localized creasing patterns are to be observed, nor whether scaleinvariance is a sufficient condition for localization.

Here, we analyze a variant of Biot's compressed elastic block, in which we replace the half-space geometry by a prism. The ridge angle $\phi$ brings in an additional parameter. Experimentally, we find buckling patterns reminiscent of creasing when the prism is flat enough $(\phi$ close to $180^{\circ}$ ), consistent with prior work 1220 . For acute enough ridge angles, however, a smooth buckling mode develops near the ridge, with a well-defined wavelength. We carry out a linear stability analysis of the compressed hyperelastic prism and investigate the competition between smooth and localizing buckling modes.

In our experiments, we use an isosceles-triangular prism made of a silicon elastomer (Ecoflex). This elas-

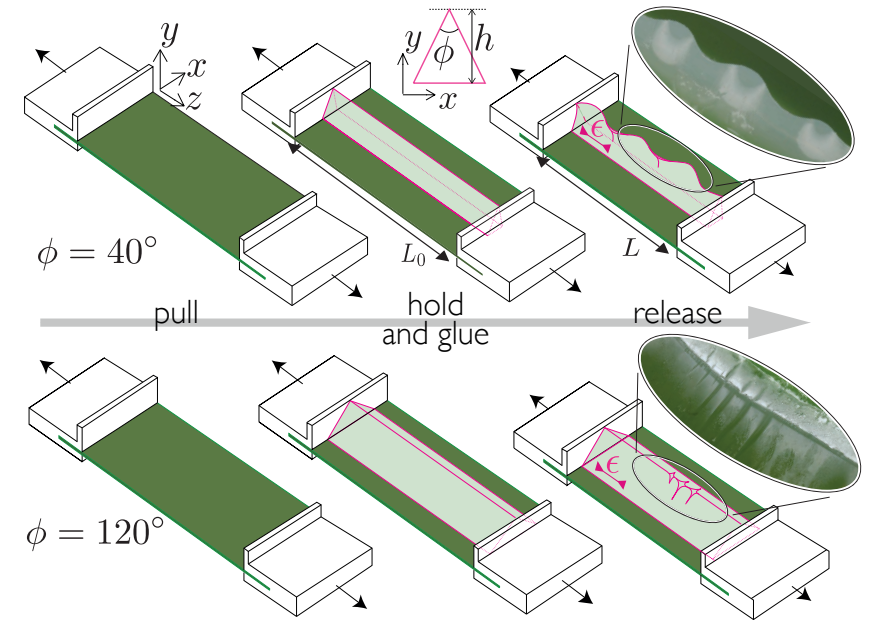

FIG. 1: Sketch of the experimental setup: to set the prism in axial compression, we first strech the substrate, then glue the prism to the substrate while keeping the substrate in tension, and finally release the substrate. This induces buckling of the prism: extended wrinkling (top row, $\phi=40^{\circ}$ ) and localized creasing (bottom row, $\phi=120^{\circ}$ ) are observed, depending on the value of $\phi$. Insets: experimental pictures.

tomer is nearly incompressible with a Young's modulus $E_{\mathrm{p}} \approx 0.06 \pm 0.02 \mathrm{MPa}$. Its lower face is bonded to a parallepipedic silicone block made of vinylpolysiloxane whose Young's modulus is $\sim 20$ times larger, $E_{\mathrm{b}} \approx$ $1.3 \pm 0.05 \mathrm{MPa}$. Both the prism and the base are obtained by casting liquid polymer into molds made of PMMA obtained by laser-cutting. We stretch the base to a length $L_{0}$ prior to glueing the prism onto it, see Fig. 1. By bringing the ends of the base closer to one another we induce a compressive axial strain $\epsilon=\frac{L_{0}-L}{L_{0}}$ in the prism, that 
depends on the current length $L<L_{0}$ of the base. At a critical value of the strain $\epsilon_{\mathrm{c}}$ an instability is observed which is localized along the free ridge of the prism, opposite to the base. Using different molds we repeat the buckling experiment for different ridge angles $\phi$ in the range $20^{\circ}$ to $120^{\circ}$. The height $h$ of the triangular prism is chosen at least ten times smaller than $L_{0}$, so we can ignore finite-length effects in the analysis.

For $\phi$ smaller than a critical value $\phi^{\star} \approx 90^{\circ}$ we observe a smooth, extended buckling mode whereby the ridge bends out of the plane of symmetry of the prism, see Fig. 2 ; ; we will refer to this as antisymmetric wrinkling (AW). For a given angle $\phi \leq \phi^{\star}$ the wavelength $\lambda$ scales close to linearly with the height of the prism $h$ for the range of heights tested in the experiments, see the inset of Fig. 2.

For $\phi \geq \phi^{\star}$ the buckling mode is entirely different, see Fig. 22: localized creases are initiated at the ridge. As the strain is increased beyond $\epsilon_{\mathrm{c}}$, more creases are formed and they spread along the lateral faces toward the base. The gap between successive creases does not appear to be regular. This buckling mode will be referred to as surface creasing (SC).

Overall, $\epsilon_{c}$ increases steadily with the ridge angle $\phi$ until it reaches a plateau at $\epsilon_{c} \approx 0.42$ for $\phi=\phi^{\star}$, where the nature of the buckling mode changes, see Fig. $2 \mathrm{~d}$. This value is lower than the critical Biot strain $\epsilon_{\text {Biot }}=$ 0.55 calculated by Biot [10, 11 for the surface instability, as explained below.

We set up a bifurcation analysis, with the aim of characterizing the instabilities and of explaining the competition between the localized and extended buckling modes. The system is modeled as an infinitely long prism with triangular cross-section $\mathcal{D}$ made of an hyperelastic material. Its elastic energy density is denoted by $W_{3 D}(\boldsymbol{E})$ where $\boldsymbol{E}=\frac{1}{2}\left(\boldsymbol{F}^{T} \cdot \boldsymbol{F}-1\right)$ is the strain tensor, $\boldsymbol{F}=\frac{\partial(x, y, z)}{\partial(X, Y, Z)}$ the transformation gradient, $(X, Y, Z)$ are the coordinates in reference configuration with $Z$ aligned with the prism axis and $Y$ along the axis of symmetry of the triangular cross-section $\mathcal{D}$, and $(x, y, z)$ are the coordinates in deformed configuration. The expression of $W_{3 D}(\boldsymbol{E})$ reflects the choice of a material law; we use a Gent model, as described in the supplementary material, with a choice of material parameters that makes this constitutive law practically equivalent to an incompressible neo-Hookean model. Working in the framework of finite elasticity, we denote by $\varphi(X, Y, Z)=(x, y, z)-(X, Y, Z)$ the displacement. The non-linear equilibrium is obtained by the principle of virtual work as

$$
\forall \widehat{\boldsymbol{\varphi}}(X, Y, Z), \int_{0}^{L_{0}} \iint_{\mathcal{D}} \boldsymbol{\Sigma}:\left(\boldsymbol{F}^{T} \cdot \widehat{\boldsymbol{F}}\right) \mathrm{d} X \mathrm{~d} Y \mathrm{~d} Z=0,
$$

where $\boldsymbol{\Sigma}=\frac{\partial W_{3 D}}{\partial \boldsymbol{E}}$ denotes the stress and $\widehat{\boldsymbol{F}}=\frac{\partial \widehat{\boldsymbol{\varphi}}}{\partial(X, Y, Z)}$ the virtual increment of deformation gradient. As the average strain $\epsilon$ is imposed by the base, we consider only ad-
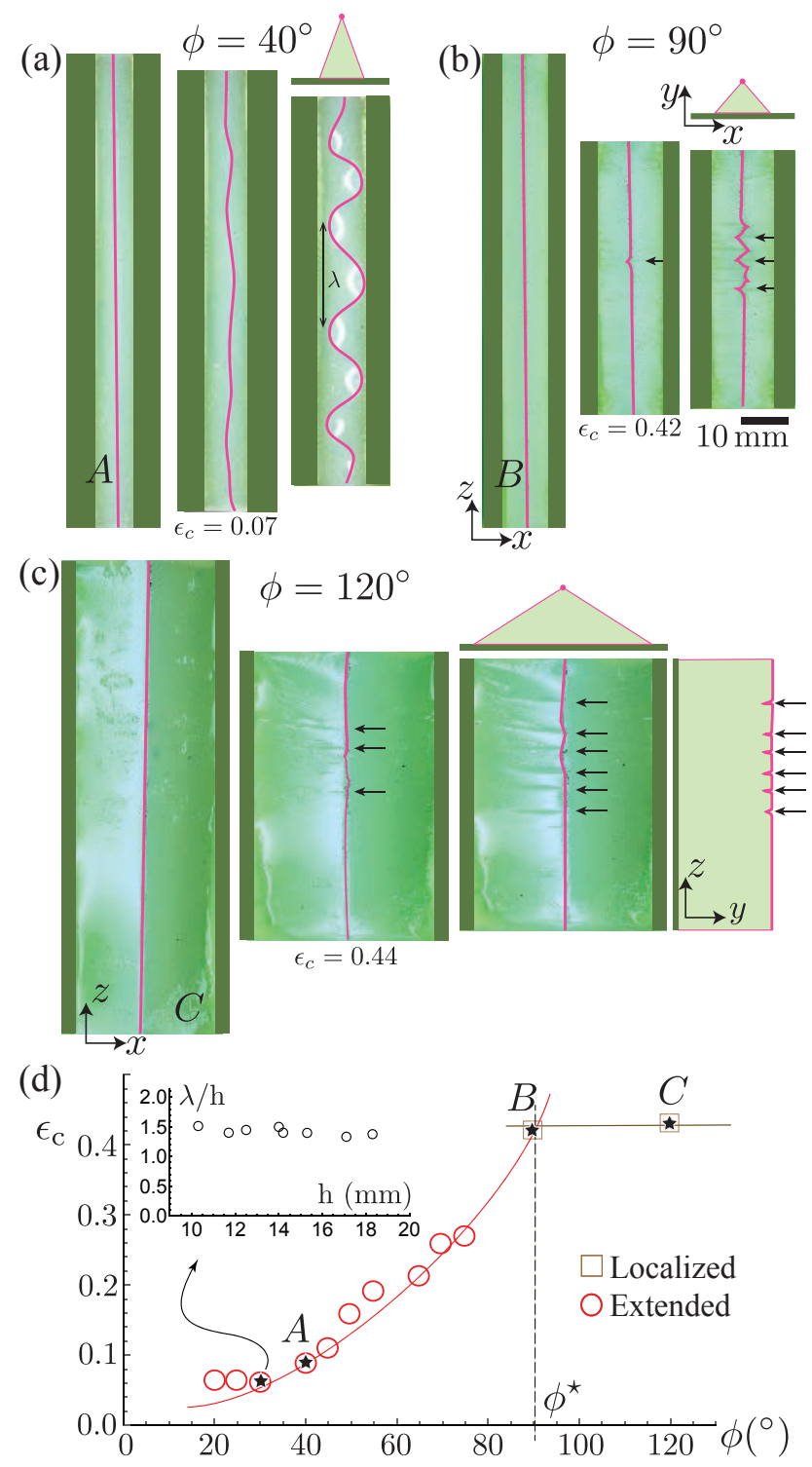

FIG. 2: Experimental results. (a-c) Top-views in the $(x, z)$ plane. Each set of pictures is for increasing axial strain $\epsilon$. A: $\phi=40^{\circ}, h=10 \mathrm{~mm}$ and $L_{0}=100 \mathrm{~mm}, \mathrm{~B}: \phi=90^{\circ}$, $h=5 \mathrm{~mm}$ and $L_{0}=100 \mathrm{~mm}$, and C: $\phi=120^{\circ}, h=10 \mathrm{~mm}$ and $L_{0}=100 \mathrm{~mm}$. Black arrows highlight the creases visible on the faces. (d) Critical strain $\epsilon_{c}(\phi)$. The thin, hand-drawn curves reveal the trend of the experimental data points. Inset: rescaled wavelength of the extended mode $\lambda / h$ for $\phi=30^{\circ}$.

missible virtual displacements $\widehat{\varphi}$ whose incremental axial strain is zero on average. Taking advantage of the fact that the buckling patterns are localized near the ridge in the experiments, we simplify the boundary conditions at the interface with the base which we replace by a free boundary.

The unbuckled solution is in a state of homogeneous 'simple' compression as described by $\varphi_{0}^{\epsilon}=\eta(\epsilon)\left(X \mathbf{e}_{x}+\right.$ $\left.Y \mathbf{e}_{y}\right)-\epsilon Z \mathbf{e}_{z}$. Here $\eta(\epsilon)$ captures the dilation of the cross-section by Poisson's effect and is found from the 
constitutive law by solving $\frac{\partial W_{3 D}}{\partial \eta}(\epsilon, \eta(\epsilon))=0$. We consider a small perturbation $\varphi_{1}$, to this invariant solution $\boldsymbol{\varphi}_{1}=\left(\xi_{x}(X, Y) \mathbf{e}_{x}+\xi_{y}(X, Y) \mathbf{e}_{y}+i \xi_{z}(X, Y) \mathbf{e}_{z}\right) e^{i q Z}$ in the form of a pure Fourier mode with wavenumber $q$. The virtual displacement $\widehat{\varphi}$ is sought in a similar form. Upon linearization and discretization using the finite-element method, the equation of equilibrium (1) takes the form

$$
\forall \widehat{\boldsymbol{\xi}}, \quad \widehat{\boldsymbol{\xi}} \cdot\left(K_{\epsilon}+q C_{\epsilon}+q^{2} M_{\epsilon}\right) \cdot \boldsymbol{\xi}_{1}=0,
$$

where $\boldsymbol{\xi}_{1}=\left(\xi_{x}, \xi_{y}, \xi_{z}\right)$ and $\hat{\boldsymbol{\xi}}=\left(\widehat{\xi}_{x}, \widehat{\xi}_{y}, \widehat{\xi}_{z}\right)$ are two vectors collecting the Fourier amplitudes of the real and virtual nodal displacements on the cross-section $\mathcal{D}$. The Fourier analysis thus yields a 2-d eigenvalue problem in which the third dimension enters through the wavenumber $q$ only. For details of this 2-d formulation and of its implementation, see Supplemental Material (Supp. Mat.) and 8 . To discretize and solve the eigenvalue problem, we make use of the finite-element library FEniCS [26] and of the SLEPc library [27].

Equation (2) is invariant when a homothety is applied to both the solution and the wavelength $2 \pi / q$; in addition, the domain $\mathcal{D}$ is scale-invariant near the tip (ridge). As a result, an infinite number of modes that are homothetic one to another appear concurrently at the critical strain $\epsilon_{\mathrm{c}}$. These modes are localized near the ridge and are associated with all possible wavenumbers: there is no selection of the wavenumber in this scale-invariant linear bifurcation analysis, see Supp. Mat. for details. By contrast, the critical strain $\epsilon_{\mathrm{c}}$ and the shape of the buckling mode (up to a dilation) are selected as a function of $\phi$.

As the unbuckled configuration is mirror-symmetric with respect to the $(y z)$ plane, the buckling modes can be either symmetric or anti-symmetric. When $\phi$ is smaller than $\approx 105^{\circ}$, the first critical buckling mode predicted by the FEM analysis is an anti-symmetric wrinkling mode (AW), see Fig. 4a. It involves lateral undulations of the ridge, see Fig. $3 \mathrm{~b}$, similar to the buckling mode seen in the experiments. The corresponding critical strain $\epsilon_{\mathrm{c}}$ is plotted in Fig. 3 a (disks) and compared to experimental results (open circles): $\epsilon_{\mathrm{c}}(\phi)$ is in good agreement with the experiments, and increases with $\phi$.

In the limit of an acute ridge angle, $\phi \rightarrow 0$, the prism can be modeled as a thin, infinitely long plate whose thickness $t(y)$ varies linearly with the distance to the ridge, $t=\phi|h-y|$. For the unbuckled solution, the midsurface of the plate is contained in the $(y z)$ plane and has an axial pre-stress $\sigma^{0}=E \epsilon$. When linearized about this solution, the Föppl-Von Kàrmàn equations for elastic plates yield, see for instance [25],

$$
\left(m_{\alpha \beta}\right)_{, \alpha \beta}+t(y) \sigma^{0} w_{, z z}=0
$$

where $w(x, y)$ is the (horizontal) deflection, $m_{\alpha \beta}=$ $D(y)\left((1-\nu) w_{, \alpha \beta}+\nu \delta_{\alpha \beta} w_{, \gamma \gamma}\right)$ denotes the bending moment, $D(y)=\frac{E t(y)^{3}}{12\left(1-\nu^{2}\right)}$ is the bending modulus of the (a)

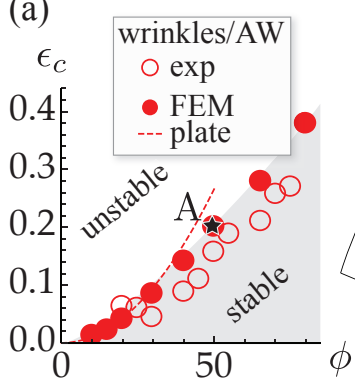

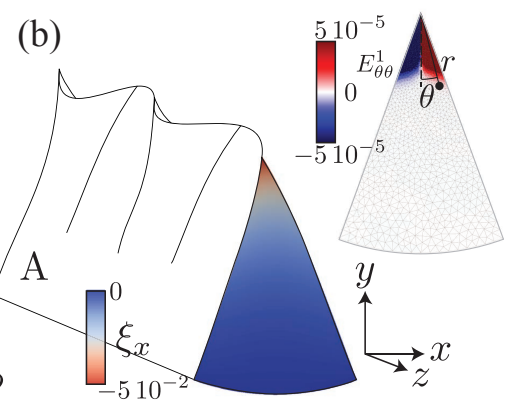

FIG. 3: Anti-symmetric wrinkling (AW). (a) Phase diagram $\epsilon_{c}(\phi)$ from experiments (open circles), simulations (disks) and analytical model (dashed curve). (b) Numerical buckling mode for $\phi=40^{\circ}, h=5 \mathrm{~mm}$, shown with an arbitrary amplitude. The two colormaps show the amplitude of the lateral displacement (left) and of the incremental hoop strain $E_{\theta \theta}^{1}$ (right).

plate, $E$ is Young's modulus and $\nu$ is Poisson's ratio. A comma in subscript denotes a partial derivative and Greek symbols are restricted to in-plane directions, $\alpha, \beta \in\{y, z\}$. We use Einstein's convention for implicit summation on repeated indices.

In the plate model, we consider perturbations that are harmonic in the axial direction and rescale the vertical coordinate using the wavelength, $w(y)=\bar{w}(q y) e^{i q z}$. When expressed in terms of $\bar{w}$ and $q$, the boundary value problem (3) and the associated boundary conditions depend on the two dimensionless parameters $\nu$ and $\bar{\sigma}^{0}=\frac{12\left(1-\nu^{2}\right) \sigma^{0}}{E \phi^{2}}$. A numerical solution based on a shooting method yields the critical value $\bar{\sigma}_{c}^{0}(\nu)$, see Supp. Mat. for details. The corresponding critical strain is $\epsilon_{\mathrm{c}}=\frac{\bar{\sigma}_{\mathrm{c}}^{0}(\nu)}{12\left(1-\nu^{2}\right)} \phi^{2}$. For our particular 3-d constitutive law, $\nu=0.45$ and we obtain $\epsilon_{\mathrm{c}}(0.45) \approx 0.35 \phi^{2}$; the dependence on Poisson's ratio is mild, $\epsilon_{\mathrm{c}}(0.50) \approx 0.33 \phi^{2}$ in the incompressible case. This prediction has no adjustable parameter and is plotted in Fig. 3 a (red dashed line): it agrees asymptotically with the finite element analysis for $\phi \rightarrow 0$. Note that $\epsilon_{c} \sim \phi^{2}$ is small when $\phi \rightarrow 0$, which is consistent with the linear elastic behavior assumed in the plate model.

Symmetric wrinkling modes (SW) are also found in the numerical bifurcation analysis. They extend on the adjacent faces on both sides of the ridge and involve an undulation of the ridge in the plane of symmetry $(y z)$, see Fig. 4 b. The strain at which the first symmetric mode appears is $\epsilon \approx 0.55$, a value which hardly depends on the ridge angle $\phi$, see Fig. 4 a. This value is consistent with the critical Biot strain $\epsilon_{\text {Biot }}=0.55$ corresponding to the existence of a marginally stable surface mode in a pre-stressed neo-Hookean half-space [10, 11. This is consistent with the fact that the SW mode is localized just beneath the faces of prism, see Fig. 4b. When $\phi$ reaches $\approx 105^{\circ}$, the critical strain $\epsilon_{\mathrm{c}}$ of the AW mode 


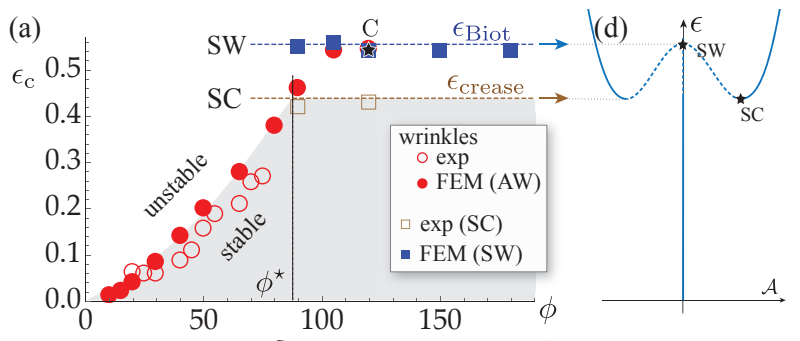

(b)

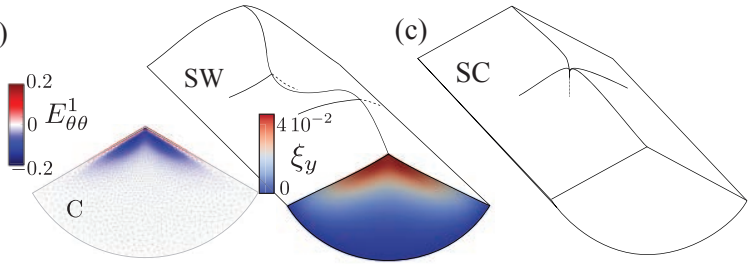

FIG. 4: (a) Full bifurcation diagram, comparing the modes predicted by the linear bifurcation analysis (AW and SW), Biot's threshold, the non-linear creasing threshold, and experiments. (b) Numerical linear buckling mode for $\phi=120^{\circ}$, $h=0.5$. Sketch of the deformed prism superimposed with a colormap of the amplitude of the vertical displacement $\xi_{y}$. Colormap of the amplitude of the hoop strain $E_{\theta \theta}^{1}$. (c) Sketch of the experimental surface creasing mode (SC) for $\phi=120^{\circ}$. (d) Sketch of the sub-critical bifurcation curve $\mathcal{A}(\epsilon)$ for creasing.

becomes larger than $\epsilon_{\text {Biot }}=0.55$ : the numerical analysis then predicts that the first buckling mode switches from an antisymmetric mode (AW) to a symmetric (SW) mode, see Fig. 4 a.

This linear analysis therefore predicts a symmetric wrinkling mode (SW) which is smooth and sinusoidal, in apparent contradiction with the localized pattern observed in the experiments. When this mode becomes unstable, all wavelengths appear concurrently: it is known that the non-linear coupling between the different wavelengths gives rise to a creasing instability through a sub-critical bifurcation [14, 21]. The buckling strain for the creasing instability in a neo-Hookean half-plane $\epsilon_{\text {crease }} \approx 0.44$ is therefore lower than that predicted by the linear analysis $\epsilon_{\text {Biot }} \approx 0.55$, see [14, 17]. Extrapolating to our problem, this suggests that our SW modes are subcritical as well, and that the critical strain $\epsilon_{\text {Biot }}$ predicted by the linear analysis needs to be corrected: the value $\epsilon_{\text {crease }}$ has been included in Fig. 4 a and indeed corresponds to the plateau observed in the experiments, see Fig. 4. Accordingly, the critical ridge angle $\phi^{\star}$ can be found by equating the critical strain for antisymmetric modes $\epsilon_{\mathrm{c}}(\phi)$ with the creasing strain $\epsilon_{\text {crease }}$ : this yields $\phi^{\star}=88^{\circ}$, see Fig. 4, which accurately matches the experimental value $\phi^{\star} \approx 90^{\circ}$.

Our linear stability analysis correctly captures the dependence of the critical strain on the ridge angle, $\epsilon_{\mathrm{c}}(\phi)$, as well as the shape of the antisymmetric mode. In our scale-free formulation, there is no selection of the wavelength. To account for the wavelength of the antisym- metric mode, one would need to consider additional ingredients in the analysis, such as subtle nonlinear effects and/or small-scale regularization. By contrast with the antisymmetric mode, the symmetric mode predicted by the linear stability analysis is not observed, as it gives rise to creasing by a subcritical bifurcation. Combining our linear analysis with the non-linear threshold for creasing, we have explained the critical value of the ridge angle $\phi^{\star} \approx 90^{\circ}$ at which the pattern changes. Interestingly, close to $\phi^{\star}$, the system displays a mix of the two behaviors: creases superimposed onto the smooth antisymmetric mode are shown in Fig. 2b, probably resulting from the non-linear interaction between the symmetric and antisymmetric modes.

The creasing localization has been explained in earlier work by non-linear coupling of the buckling modes. A remarkable finding of our experiments is that our system features both localized creases and a smooth extended buckling pattern: the coupling between modes of different wavelengths is effective for the symmetric mode (leading to creases) but it is not effective for the antisymmetric mode, surprisingly. Therefore, scale-invariance in not a sufficient condition for localization and the exact conditions in which modes of different wavelengths can cooperate remain to be elucidated: the compressed hyperelastic prism provides a workbench for future non-linear analyses of creasing.

Acknowledgments: We thank A. El Ouardy for his contribution to the experiments. CM acknowledges the financial support of Project ANR-13-JS09-0009 (Agence Nationale de la Recherche, 2014).

[1] T. Savin, N. A. Kurpios, A. E. Shyer, P. Florescu, H. Liang, L. Mahadevan, and C. J. Tabin, Nature 476, 57 (2011).

[2] M. A. Biot, Proceedings of the Royal Society A: Mathematical, Physical and Engineering Sciences 242, 444 (1957).

[3] E. Sultan and A. Boudaoud, J. Appl. Mech 75, 1002 (2008).

[4] Y. Cao and J. W. Hutchinson, Journal of Applied Mechanics 79, 1019 (2012).

[5] D. Lee, N. Triantafyllidis, J. R. Barber, and M. D. Thouless, Journal of the Mechanics and Physics of Solids 56, 858 (2008).

[6] J. Huang, J. Liu, B. Kroll, K. Bertoldi, and D. R. Clarke, Soft Matter 8, 6291 (2012).

[7] J. Liu, J. Huang, T. Su, K. Bertoldi, and D. Clarke, PLoS ONE 9, e93183 (2014).

[8] C. Lestringant and B. Audoly, Journal of the Mechanics and Physics of Solids (2016).

[9] D. Terwagne, M. Brojan, and P. M. Reis, Advanced materials 26, 6608 (2014).

[10] M. A. Biot, Proceedings of the Royal Society A: Mathematical, Physical and Engineering Sciences 273, 329 (1963). 
[11] M. A. Biot, Mechanics of Incremental Deformations (Wiley, 1965).

[12] W. Hong, X. Zhao, and Z. Suo, Applied Physics Letters 95 (2009).

[13] J. Yoon, J. Kim, and R. C. Hayward, Soft Matter 6, 5807 (2010).

[14] E. Hohlfeld and L. Mahadevan, Physical Review Letters 106, 105702 (2011).

[15] S. Mora, M. Abkarian, H. Tabuteau, and Y. Pomeau, Soft Matter 7, 10612 (2011).

[16] E. Hohlfeld and L. Mahadevan, Physical Review Letters 109, 025701 (2012).

[17] S. Cai, D. Chen, Z. Suo, and R. C. Hayward, Soft Matter Communication 8, 1301 (2012).

[18] L. Jin, D. Chen, R. C. Hayward, and Z. Suo, Soft Matter 10, 303 (2014).

[19] L. Jin, A. Auguste, R. C. Hayward, and Z. Suo, Journal of Applied Mechanics 82 (2015).

[20] L. Jin and Z. Suo, Journal of the Mechanics and Physics of Solids 74, 68 (2015).

[21] Y. Cao and J. W. Hutchinson, Proceedings of the Royal Society A: Mathematical, Physical and Engineering Sciences (2011).

[22] Y. B. Fu and P. Ciarletta, Proceedings of the Royal Soci- ety of London A: Mathematical, Physical and Engineering Sciences 471 (2015).

[26] K. Ølgaard, A. Logg, and G. Wells, SIAM Journal of scientific computing 31, 849 (2009).

[27] V. Hernandez, J. E. Roman, and V. Vidal, ACM Trans. Math. Software 31, 351 (2005).

[25] B. Audoly and Y. Pomeau, Elasticity and geometry: from hair curls to the nonlinear response of shells (Oxford University Press, 2010).

[26] K. Ølgaard, A. Logg, and G. Wells, SIAM Journal of scientific computing 31, 849 (2009).

[27] V. Hernandez, J. E. Roman, and V. Vidal, ACM Trans. Math. Software 31, 351 (2005).

[31] F. Tisseur and K. Meerbergen, SIAM Review 43, 235 (2001).

[32] Y. Saad, Numerical methods for large eigenvalue problems (SIAM, Philadelphia, 2011).

[30] See Supplemental Material [url], which includes Refs. 31, 32.

[31] F. Tisseur and K. Meerbergen, SIAM Review 43, 235 (2001).

[32] Y. Saad, Numerical methods for large eigenvalue problems (SIAM, Philadelphia, 2011). 